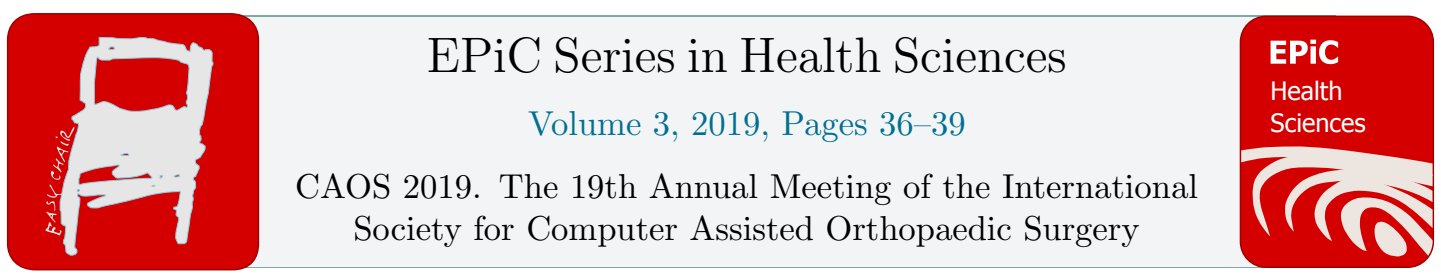

\title{
A Robotic Nondestructive Osteochondral Tissue Harvesting for Autograft Transplantation
}

\author{
Pradipta Biswas, Sakura Sikander, Pankaj Kulkarni, and Sang-Eun Song* \\ Department of Mechanical and Aerospace Engineering, University of Central Florida, USA \\ pvbiswas@knights.ucf.edu, sakura@knights.ucf.edu, pankajpkulkarni@knights.ucf.edu, \\ s.song@ucf.edu
}

\begin{abstract}
Osteoarthritis is the degeneration of bone-cartilage. Healthy cartilage absorbs mechanical stress and provides smooth limb movement. Cartilage has poor healing capabilities due to the absence of blood, lymphoid tissue, and nerve that makes treatment of the damaged cartilage difficult, making surgical intervention an inevitable solution. Mosaicplasty is a popular surgical practice involving transplantation of small cylindrical bone-cartilage plugs to refill the lesion. A lack of custom-shaped donor harvesting mechanism makes it impossible to fill the lesion with a single graft. The success of transplanting a customized autograft to replace the osteochondral lesion lies in effective extraction of the autograft from the donor site. Currently, no method exists to harvest such grafts since it requires access to the root side of donor. In this paper, we propose a robotic cartilage-bone removal mechanism to harvest a custom-shaped autograft. Our method involves drilling a profile determined from the lesion to be removed and slicing off the desired cartilage-bone graft from the root. We designed a new graft removal mechanism capable of inserting a thin wire saw and slicing through the root of the prepared profile to extract an intact autograft. The device can be attached to a standard 6DOF robotic arm that can provide profile drilling and gross positioning of the graft removal device.
\end{abstract}

\section{Introduction}

Nearly 27 million people aged 25 or above in the United States are affected by osteoarthritis (OA) each year [1]. OA is the deterioration of articular cartilage and subchondral bone which is responsible for fluid and frictionless movement among joints and absorbs shock. Degeneration of this cartilage often causes pain, joint stiffness, and disability [2].

\footnotetext{
${ }^{*}$ Corresponding author
} 
Post traumatic osteoarthritis (PTOA) develops after a joint injury and affects 5.6 million people each year causing $12 \%$ osteoarthritis in hip, knee, and ankle [3]. Initial injuries from fracture, cartilage damage, acute ligament sprain or chronic ligamentous instability can be the cause of PTOA. Mild PTOA can be treated with weight loss, low impact exercise, and non-steroidal anti-inflammatory medications. While these treatments will provide symptomatic reliefs, they will not reverse the cartilage damage. Bones are highly vascularized tissue with self-healing capabilities. Cartilage, however, lacks blood vessels, lymphoid tissue, and nerve that leads to poor self-healing capabilities [4]. Therefore, surgical intervention becomes a necessary procedure.

Microfracture, autologous osteochondral cylinder transplantation (mosaicplasty), osteochondral allograft transplantation, artificial bone graft substitutes, and cell-based repair techniques such as autologous chondrocyte implantation (ACI) are the common methods used to treat a lesion [5]-[7]. Microfracture is commonly used for small areas and other methods listed above can be used for larger areas. ACI is a more expensive procedure compared to articular bone-cartilage grafting and requires two sub-procedures. For osteochondral transplantation, plugs of healthy cartilage with subchondral bone are extracted either from the joints of a cadaver (allografts) or from a non-load bearing area of the patient in the shape of cylinders (as in case of mosaicplasty) and inserted into the arthritic defect as a whole or in mosaic manner (autografting) respectively.

Due to current technological limitations, harvesting large grafts is not practical. Harvesting a larger and custom-shaped graft for the damaged osteochondral area requires effective extraction of the graft from the donor site. Currently, no method exists to execute custom-shaped donor harvesting because of the complexity and precision needed to access and extract the graft from its root. To overcome the limitation, we designed a novel robotic mechanism to perform customized graft extraction from the donor site for autografting.

\section{Materials and Methods}

A 6 degree of freedom (DOF) robotic arm and a specially designed tool is used in our approach. First, a contour is prepared around the desired donor site using a drilling tool mounted on the robotic arm with the assistance of a surgeon. Then, a modified Gigli saw type of approach is taken to slice off a desired shaped graft from the donor site. As shown in Fig. 1(a) for the harvesting tool a Gigli saw type flexible bone cutting wire passes through two guides placed vertically between the contours created around the donor site. The flexible saw oscillates through the guides and slices the bottom layer of the

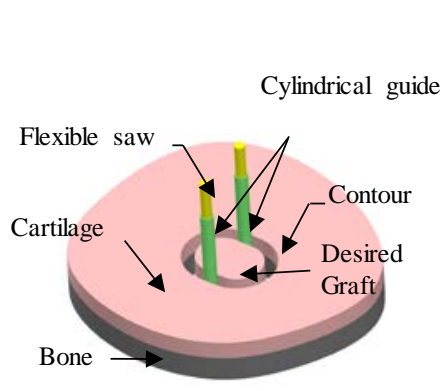

(a)

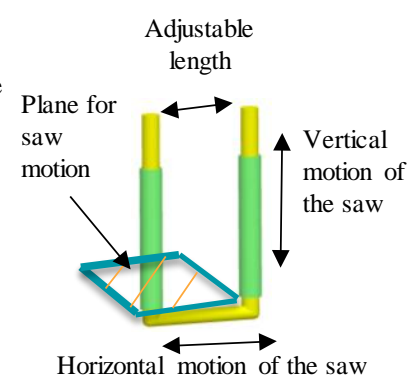

(b)

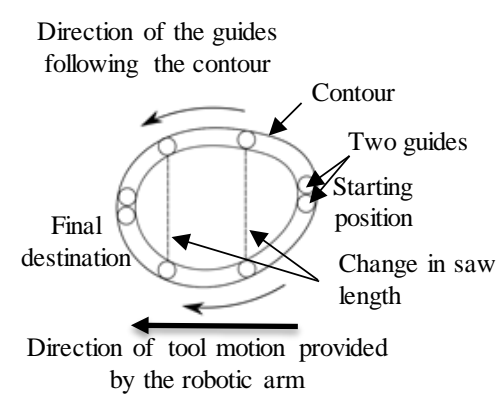

(c)

Figure 1: (a) Two guides inserted in the contour along with the flexible saw for graft harvesting, (b) Conversion of the flexible saw motion to the cutting plane, and (c) Top view of the guides following the contour starting from its initial position to its final destination while the robotic arm is providing the tool motion for slicing the graft from its base. 


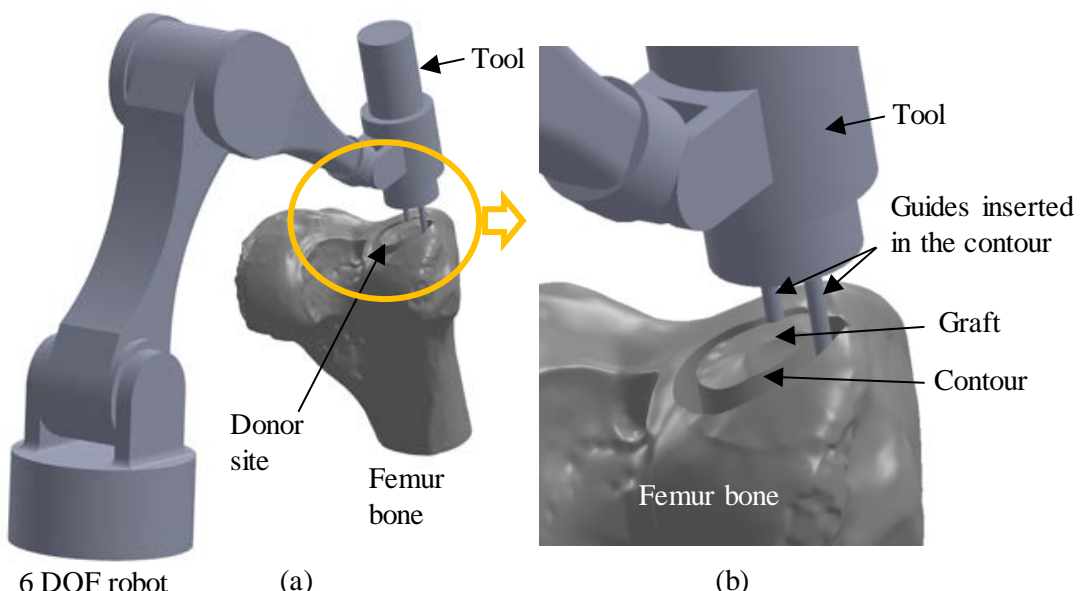

Figure 2: (a) Over all setup using the 6-DOF robotic arm (b) Schematic of the position of the tool guide while the robotic arm is assisting with the slicing motion

graft base. The transverse Gigli saw motion is converted to the motion along the coronal plane for slicing the graft from the base (Fig. 1(b)). An existing 6 DOF robotic arm is utilized and acts as the gross positioning system (GPS) for the tool which is mounted on its end effector (Fig. 2(a) and (b)). Once the two guides are placed in the contour, the robotic arm will provide the desired motion to the tool to follow the contour and simultaneously the guides will change the distance between them while moving through the contour (Fig. 1(c)). The tool provides the mechanism for the linear motion of the guides and the ability to change the length of the flexible saw while following the contour.

\section{Results}

We have designed a CAD model and a mechanism for a robotically operated tool to extract a personalized graft from a donor site for autografting. The tool utilizes a flexible saw and converts the cutting motion from the transverse plane to the coronal plane. A 6-DOF robotic arm provides the gross positioning to the tool in terms of depth control and a planar motion for slicing the graft. The two guides are capable of sliding through the contour by changing the linear distance between them and adjusting the flexible saw length accordingly. With the combination of the robotic arm motion and varying the distance between the two guides it is possible to follow a desired profile and slice off a custom sized graft from its base.

\section{Discussion}

We have developed a robotically operated method and mechanism for harvesting a bone-cartilage graft of any customized shape from a donor site for autografting. Currently, no method exists to extract the custom shaped bone-cartilage graft from its base. Our approach can enhance current surgical treatments for PTOA. This method and mechanism can be utilized to extract bone-cartilage graft of any size. Since, extracting a custom shaped graft in a tight spaced donor site requires precision, therefore the robotics assisted bone-cartilage harvesting will be able to enhance the performance of the surgical 
procedure. Future work will include developing a prototype of the system and performing an experiment on sawbone using the 6 DOF robotic arm.

\section{References}

[1] R. C. Lawrence et al., "Estimates of the prevalence of arthritis and other rheumatic conditions in the United States. Part II.,” Arthritis Rheum., vol. 58, no. 1, pp. 26-35, Jan. 2008.

[2] A. C. Thomas, T. Hubbard-Turner, E. A. Wikstrom, and R. M. Palmieri-Smith, "Epidemiology of Posttraumatic Osteoarthritis,” J. Athl. Train., vol. 52, no. 6, pp. 491-496, Jun. 2017.

[3] T. D. Brown, R. C. Johnston, C. L. Saltzman, J. L. Marsh, and J. A. Buckwalter, "Posttraumatic Osteoarthritis: A First Estimate of Incidence, Prevalence, and Burden of Disease,” J. Orthop. Trauma, vol. 20, no. 10, pp. 739-744, Nov. 2006.

[4] Y. X. Lin et al., "In vitro and in vivo evaluation of the developed PLGA/HAp/Zein scaffolds for bone-cartilage interface regeneration.,” Biomed. Environ. Sci., vol. 28, no. 1, pp. 1-12, Jan. 2015.

[5] B. Kinner and M. Spector, "Cartilage-current applications," Orthop. tissue Eng. basic Sci. Pract. Dekker, New York, NY, 2004.

[6] R. S. Tuan, "Experimental Principles and Future Perspectives of Skeletal Tissue Engineering," Tissue Eng. Musculoskelet. Clin. Pract. Ed. Rosemont, Am. Acad. Orthop. Surg., 2004.

[7] J. D. Harris, R. A. Siston, X. Pan, and D. C. Flanigan, “Autologous Chondrocyte Implantation,” J. Bone Jt. Surgery-American Vol., vol. 92, no. 12, pp. 2220-2233, Sep. 2010. 